\title{
Raman selection rules in the presence of an electric field gradient
}

\author{
C. L. Jahncke, ${ }^{*}$ E. J. Ayars** and H. D. Hallen*** \\ * Physics Department, St. Lawrence University, Canton, NY 13617 \\ ** Physics Department, Walla Walla College, College Place, WA 99324 \\ *** Physics Department, North Carolina State University, Raleigh, NC 27695-8202
}

The metal aperture at the apex of a near-field scanning optical microscope (NSOM) probe locally concentrates the electric field. As these evanescent fields decay on a nanometer length-scale, strong field gradients are produced. These gradients have profound effects on the Raman spectra of samples within them, leading to a "Gradient-Field Raman" effect. It leads to new selection rules for surface enhanced Raman spectroscopy (SERS), for example see [1,2] and references within, and also to differences between far-field and near-field Raman spectroscopy measured with a near-field optical microscope. [3] We describe how a strong gradient of the electric field can alter the Raman spectra, and investigate its implications on selection rules. Heuristically, the field gradient causes the Coulomb force on a polarized bond to vary during the vibration, providing a new coupling mechanism between the field and the vibration. These selection rules differ markedly from the usual Raman selection rules, and allow Raman-like observation of strong IR (not normally Raman) vibrations.

Most of the Raman work (except NSOM) performed near metal surfaces has been concerned with SERS. We do not comment on SERS models here, but note which peaks not normally seen in Raman are expressed. We discuss elsewhere [4] the SERS work on benzene, several cyanides, and $\mathrm{C}_{60}$. Here we concentrate on pyrazine, which is a benzene ring with two opposing carbons replaced with $\mathrm{N}$. It has been studied extensively since it is complicated enough to have several vibration modes (with $\mathrm{D}_{2 \mathrm{~h}}$ symmetry), but simple enough that the bulk vibrations are either Raman or IR active, but not both. It has been studied on $\mathrm{Ni}, \mathrm{Cu}, \mathrm{Au}$, and $\mathrm{Ag}$. [5-7] When shifts of the usual Raman lines between bulk or solution and surface Raman are used as a gauge of substrate interaction, the pyrazine reacts most with $\mathrm{Ni}$, then $\mathrm{Au}$ and $\mathrm{Cu}$, and least with $\mathrm{Ag}$. [5] We therefore concentrate on the $\mathrm{Ag}$ results. The strong IR line at $1484 \mathrm{~cm}^{-1}$ is the strongest new line in the various papers, but with intensity comparable to the normally allowed modes in some studies $[6,7]$, and much reduced from them in others. [5] Another strong IR line at $1418 \mathrm{~cm}^{-1}$ is often nearly as strong as the $1484 \mathrm{~cm}^{-1}$ line, $[5,7]$ but absent in other studies [6] and for studies involving the strongly interacting metals. [5] The strong IR - strong new Raman lines analogy for GFR holds, but the irreproducibility is troublesome. Perhaps the orientation of the molecules, which matters in GFR, depends strongly on sample preparation. The intensity of these lines is sensitive to electrochemical potential, [7] and the orientation of pyrazine on $\mathrm{Ni}$ does change with concentration, lying flat at low concentrations and on-end at higher concentrations. [5] This could explain why these in-plane (so not GFR affected for flat orientation) vibrations are not completely reproducible.

In NSOM, a sharpened optical fiber is coated with aluminum to form an aperture. The probe is positioned near the surface under lateral force feedback. The NSOM is used in illumination mode, with $514 \mathrm{~nm}$ Ar ion laser light coupled into the fiber probe. Reflected light is collimated with a 0.50 NA lens, passed through a holographic filter, focused into a Czerny-Turner spectrometer, and finally collected onto a cooled ( $-45 \mathrm{C}$ ) CCD camera. A near field spectra of KTP is shown in Fig. 1. The general shape is typical of the spectra at various distances from the sample. This region of the Raman spectrum contains vibrations primarily from $\mathrm{TiO}_{6}$ stretching modes in the KTP. The near-field peak shown in green in figure 1 is the strong totally symmetric, A1, vibration mode, which has been observed before in both near- and far-field measurements. [3] The changes observed as the probe approaches the surface are rather small, so we resort to comparative spectra. Several spectra taken far from the surface were averaged and subtracted from single spectra acquired closer to the surface. Two peaks at different energies than the original peaks are observed: the B1 peak (not allowed in the 
geometry of our far-field experiment) is visible in figure 1 (pink) while the strong IR absorption mode at $712 \mathrm{~cm}^{-1}$ [3] is not. The probe-sample distance dependence of the two peaks is similar, and that of the B1 peak is shown in Fig. 2, along with the best-fit Raman and GFR models. The GFR describes the data quite well except for the derivative-like variation near $90 \mathrm{~nm}$, which we attribute to coupling with plasmons on the Al probe coating. [8]

In summary, we have described a new mechanism by which a strong gradient of the electric field can cause normally forbidden vibration modes to appear in Raman spectra. The amplitude of the signal should be similar to that of the allowed Raman modes, and the relative strength of the modes should be similar to those in infrared spectroscopy, for IR allowed modes. Since infrared and Raman modes are complementary in many materials, this gradient-field Raman spectroscopy should help to provide a full vibrational analysis of a sample in a single measurement, especially when combined with an NSOM measurement, which allows the GFR terms to be preferentially reduced for positive identification of modes. The GFR effect is strongly dependent on the tip-sample distance. This results in a preferential sensitivity to surface rather than bulk effects, and raises the possibility of measuring Raman shifts of the surface, where the vibration levels inherently differ from the bulk. [9]

\section{References}

[1] Martin Moskovits, Rev. Mod. Phys. 57, 783 (1985).

[2] J. A. Creighton, "The selection rules for surface-enhanced Raman spectroscopy," in Spectroscopy of Surfaces, Edited by R. J. H. Clark and R. E. Hester (John Wiley \& Sons, New York, 1988) 37.

[3] C. L. Jahncke, H. D. Hallen and M. A. Paesler, J. of Raman Spectroscopy, 27, 579 (1996).

[4] E. J. Ayars, H. D. Hallen and C. L. Jahncke, "Electric field gradient effects in Raman spectroscopy," Phys. Rev. Lett. 85, 4180 (2000).

[5] Q.J. Huang, J.L. Yao, B.W. Mao, R.A. Gu and Z.Q. Tian, Chem. Phys. Lett. 271, 101 (1997).

[6] Gayle R. Erdheim, Ranald L. Birke and John R. Lombardi, Chem. Phys. Lett. 69, 495 (1980).

[7] Ralf Dornhaus, Marshall B. Long, Robert E. Benner and Richard K. Chang, Surface Science, 93, 240 (1980).

[8] H. D. Hallen and E. J. Ayars, (in preparation).

[9] This work was supported by the National Science Foundation through grant DMR-9975543 and the Research Corporation through grant CC5342.

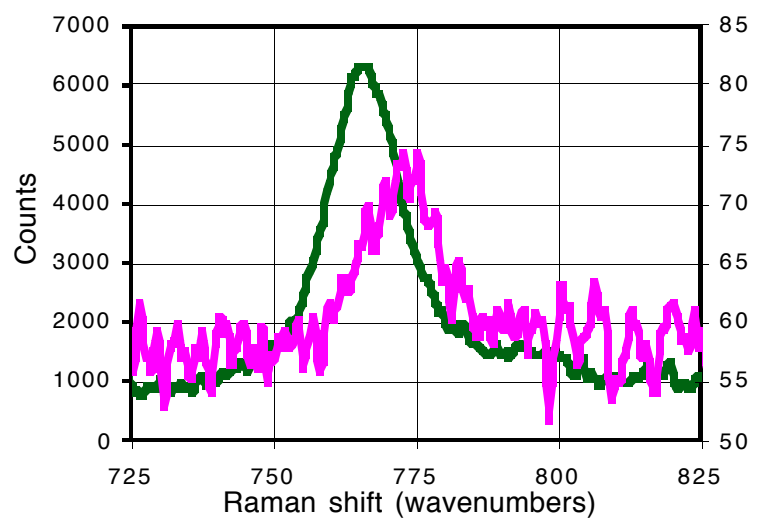

FIG. 1. A NSOM-Raman spectra taken with the probe nearly in contact with the surface, (green, left axis) is compared to a near-far difference spectra. (pink, right axis)

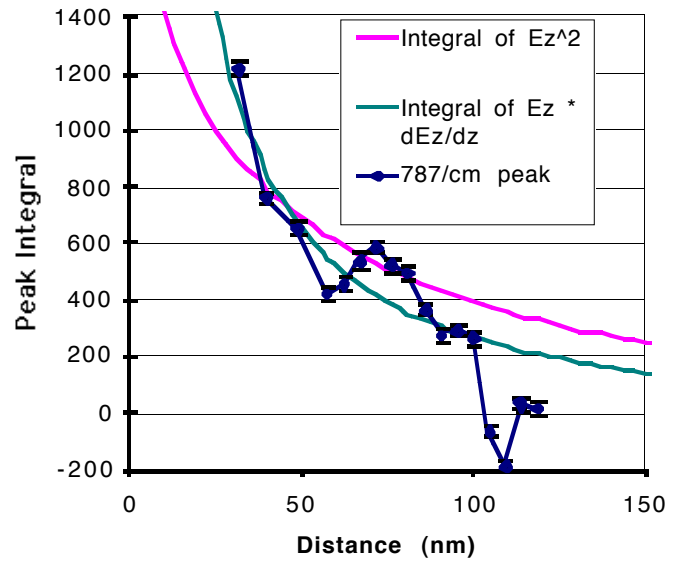

FIG. 2. The probe-sample distancedependence of the NSOM-Raman difference spectra is compared to the standard Raman and GFR models. 